\title{
Utilização da densitometria óssea como método de avaliação dos resultados da utilização de BMP bovina em artrodese de coluna em coelhos
}

\author{
Bone densitometry in the evaluation of the results obtained with the use of bovine BMP \\ in spine arthrodesis in rabbits
}

Paulo Roberto Moura Machado ${ }^{1 *}$, Eduardo Barros Puertas ${ }^{2}$, Eulázio Taga ${ }^{3}$, Nilson Nonose 4

\section{RESUMO}

As BMPs, proteínas indutoras de crescimento ósseo, desde o início de sua utilização, têm sido avaliadas em diferentes modelos experimentais objetivando determinar sua eficácia, sendo necessário o estabelecimento de métodos de avaliação que permitam melhor compreensão dos resultados.

Objetivo: Este estudo tem por objetivo avaliar a utilização da densitometria óssea como método de avaliação do incremento ósseo observado quando da utilização de BMP bovina em artrodeses intertransversas da coluna lombar de coelhos. Métodos: Foram utilizados dois grupos de dez coelhos neozelandeses fêmeas, submetidos a artrodese intertransversa da coluna lombar, segmento L5/L6, por via posterior. No primeiro grupo foi utilizado enxerto homólogo e no segundo a associação de enxerto homólogo com biocomposto (BMP bovino, 1,0 mg e hidroxiapatita, 9,0 mg). Os animais foram acompanhados por 15 semanas, isolados em cativeiro e avaliados diariamente por veterinário sob o ponto de vista clínico e neurológico e, posteriormente, submetidos à realização de densitometria óssea por tomografia computadorizada. Resultados: Foram obtidas 268 medidas de densidade óssea do osso normal, 134 medidas do osso neoformado de enxerto homólogo isolado e 134 medidas do osso neoformado pela associação enxerto homólogo e BMP. Esses valores foram submetidos à análise estatística que demonstrou incremento ósseo significativo $(p=0,034)$ do grupo BMP/HAP, quando comparado ao grupo controle. Os valores de densidade óssea do osso normal foram submetidos à mesma análise estatística onde se encontrou resultado semelhante. Conclusão: A densitometria óssea realizada pela tomografia computadorizada apresenta-se como método alternativo para avaliação de resultados quando utilizado BMP em estudos experimentais. Estudos subseqüentes deverão ser realizados para melhor entendimento da variação de densidade óssea encontrada quando da comparação das medidas do osso normal nos dois grupos.

Descritores: Proteína morfogenética óssea; Transplante ósseo; Durapatita; Densidade óssea; Tomografia computadorizada espiral.

\section{SUMMARY}

The object of this study is to evaluate the use of the bone densitometry as an evaluation method of the bone increment observed when we used bovine BMP in intertransverse arthrodesis of the rabbits' lumbar spine. Since the discovery of the BMP bone inductive properties, countless experimental models have been proposed. This caused the need of setting up evaluation methods to make possible a better understanding of the found results. Twenty female rabbits from New Zealand, divided in two groups, were submitted to the intertransverse arthrodesis of the lumbar column, segment L5/L6, posterior side. In the first group it was used autologous graft and in the second an association of autologous graft with biocompound (bovine BMP, $1.0 \mathrm{mg}$ and hydroxyapatite, $9.0 \mathrm{mg}$ ). The animals were maintained in captivity, isolated and after 15 weeks submitted to the execution of bone densitometry by computerized tomography. 268 bone density measures of the normal bone, 134 measures of the newly formed bone by the association of the isolated autologous graft and 134 measures of the newly formed bone by the association autologous graft and BMP were obtained, what demonstrated a significant bone increment after statistical analysis $(p=0.034)$ of the BMP/HAP group, when compared to the control group. We found this same variation of bone density analyzing the normal bone. The bone densitometry accomplished by the computerized tomography is an alternative method to assess the results when the BMP is used in experimental studies. Further studies should be accomplished for better understanding of the bone density variation found when the measures of the normal bone in the two groups are compared.

Keywords: Bone morphogenetic protein (BMP); Bone transplantation; Durapatite; Bone density; Tomography.
Trábalho realizado na Universidade Federal de São Paulo UNIFESP.

1 - Pós-graduando do Programa de Pós-graduação em Ortopedia e Traumatologia.

2 - Professor Adjunto - Livre Docente, Chefe do Grupo de Patologias da Coluna Vertebral da Disciplina de Ortopedia do Departamento de Ortopedia e Traumatologia

3 - Professor Titular da Faculdade de Odontologia de Bauru da Universidade de São Paulo (USP)

4 - Médico Residente do Serviço de Ortopedia e Traumatologia da Universidade São Francisco - Bragança Paulista.
Endereço para corrêspondencia: Rua Santa Clara, 525 - 12900-470 - Bragança Paulista - SP E-mail: paulormm@terra.com.br 


\section{INTRODUÇÃO}

Desde os primeiros relatos de enxertos ósseos realizados, vários materiais têm sido apresentados como alternativas para enxertos ósseos, tornando assim, necessária a realização de estudos específicos para a demonstração de resultados, com a utilização de enxertos não autólogos na coluna vertebral.

Inúmeras associações entre substâncias osteoindutoras e diferentes carreadores têm sido apresentadas na literatura, bem como a escolha de um modelo experimental pode variar de acordo com o objeto de estudo.

Alguns métodos de avaliação como palpação manual, radiografias simples, tomografia computadorizada, estudos histológicos e ensaios biomecânicos são freqüentemente utilizados, apresentando resultados valiosos e elucidando alternativas para o uso das BMPs na prática clínica.

\section{MATERIAL E MÉTODOS}

Vinte coelhos brancos, adultos, fêmeas, com peso variando de 2,0 a 2,5 kg e aproximadamente um ano de idade, foram mantidos em biotério, isolados e alimentados com dieta "ad libitum", 100 gramas diárias de ração para coelhos e água. Foram inspecionados sob o ponto de vista clínico e neurológico diariamente.

Foram formados dois grupos de dez animais, sendo o primeiro grupo chamado controle, onde fora realizado procedimento cirúrgico, sob anestesia dissociativa, utilizado para tal, 2,5 $\mathrm{mg}$ de quetamina e $1 \mathrm{mg}$ de xylazina, diluídos em 0,1 ml de água destilada, para cada 100 gramas de peso do animal, administrados por via intramuscular profunda, sendo observado tempo de latência variável de 30 a 40 minutos. Após observar-se ausência de reflexos à dor, fora realizada tricotomia da região lombar, com área aproximada de $15 \mathrm{~cm}$ quadrados, objetivando a porção distal da coluna lombar (segmento L5-L6).

Os animais foram então conduzidos à sala de cirurgia e submetidos à assepsia de pele com povidine degermante e clorexidine.

Em seguida, foram submetidos à incisão mediana posterior, dissecados os processos espinhosos e as lâminas até evidenciar os processos transversos de L5 e L6, que então eram decorticados e preparados para colocação do enxerto.

Foi obtido previamente enxerto homólogo de coelho doador, realizado após anestesia geral e eutanásia, onde foram retirados, seguindo normas assépticas, segmentos de ossos longos (fêmures e tíbias), sendo então fragmentados e colocados nos sítios receptores, volume de $3 \mathrm{ml}$ de enxerto de cada lado das transversas previamente preparadas.

Em seguida, realizou-se a sutura por planos, musculatura e pele, com fio de nylon 3.0.

O segundo grupo de animais foi submetido ao mesmo procedimento descrito anteriormente, porém foi associado ao enxerto homólogo a mistura BMP-Hidroxiapatita na seguinte composição: 0,25 cc de

biomaterial, composto de 9,0 mg de hidroxiapatita pura e 1,0 mg de BMP.

Todos os procedimentos realizados foram submetidos a apreciação da Comissão de Ética da Universidade Federal de São Paulo - UNIFESP - EPM.

Após os procedimentos, os animais foram então mantidos em cativeiro, isolados e alimentados com dieta "ad libitum" e água por um período de 15 semanas.

Durante o seguimento, um animal de cada grupo apresentou quadro de déficit neurológico progressivo de membros inferiores, evoluindo para paraplegia total na quarta semana de seguimento pós operatório. Apresentavam ainda episódios de infecção urinária, sendo assim excluído do experimento. Não foram observadas outras complicações.

Os animais foram então, submetidos à realização de tomografias computadorizadas "multislices", sendo utilizado apareIho modelo SYNERGY, fabricante GE MEDICAL SYSTEMS, seguindo os seguintes parâmetros: 10cm DFOV, 100kv, 150mA, $3 \mathrm{~mm}$ gap e $1 \mathrm{mmslice}$.

Cada animal foi anestesiado, conforme técnica já descrita anteriormente e submetido à realização de cortes tomográficos do segmento lombar.

Após realização de imagem em perfil (scalt), foram submetidos a cinco cortes tomográficos, com intervalos de $3 \mathrm{~mm}$ entre cada corte, objetivando avaliar o segmento L5-L6.

Para cada um dos cortes foram re-

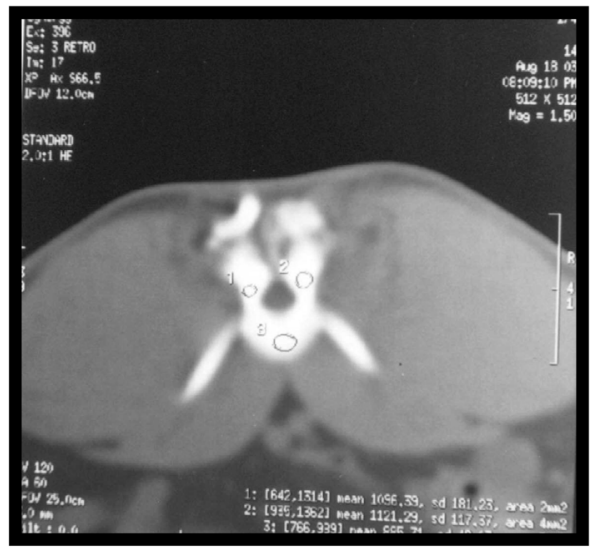

Figura 1- Locais referidos para medidas da densidade óssea no osso normal.

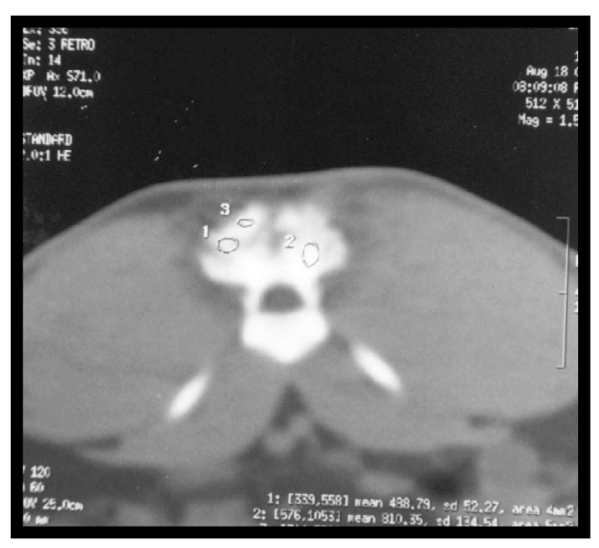

Figura 2 -Locais de medida de densidade óssea na área de enxerto. alizadas três medidas de densidade mineral óssea da vértebra normal e do osso neoformado, segundo a técnica radiológica de Hounsfield ${ }^{(8)}$, para ambos os grupos, (Figuras 1 e 2).

Sendo assim, foram obtidos 268 medidas de densidade óssea do osso normal da espécie utilizada como modelo experimental, 134 medidas de densidade mineral óssea do osso neoformado, quando da utilização de enxerto homólogo de doador isolado e 134 medidas de densidade mineral óssea do osso neoformado quando utilizada a associação de enxerto homólogo de doador associado ao composto BMP-HAP.

\section{RESULTADOS}

Os valores densitométricos obtidos, em unidades Hounsfield, estão demonstrados nas tabelas a seguir: (Tabelas 1 e 2)

Os resultados foram então avaliados estatisticamente e estão descritos a seguir.

\section{ANÁLISE ESTATÍSTICA}

Para comparação das respostas dos grupos foi utilizado o modelo de análise de variância com medidas repetidas com dois fatores: grupo e local. Os resultados demonstraram que os grupos não se diferenciam quando comparadas a diferença entre a res- 


\begin{tabular}{|c|c|c|c|c|c|c|c|}
\hline & & \multicolumn{3}{|c|}{ OSSO NORMAL } & \multicolumn{3}{|c|}{ ENXERTO } \\
\hline \multirow{5}{*}{$\begin{array}{l}\stackrel{0}{\Xi} \\
\bar{\Xi} \\
\stackrel{0}{0}\end{array}$} & Amostra 1 & 647,85 & 817,98 & 881,29 & 229,79 & 212,33 & 212,66 \\
\hline & Amostra 2 & 736,03 & 907,67 & 904,96 & 838,82 & 280,00 & 752,15 \\
\hline & Amostra 3 & 817,07 & 761,53 & 918,90 & 538,27 & 622,11 & 652,09 \\
\hline & Amostra 4 & 624,94 & 729,94 & 367,72 & 390,47 & 516,77 & 434,45 \\
\hline & Amostra 5 & 985,50 & 706,00 & 829,20 & 462,10 & 549,22 & 144,95 \\
\hline \multirow{5}{*}{$\begin{array}{l}\text { N } \\
\stackrel{0}{ } \\
\overline{\bar{\Xi}} \\
\stackrel{0}{0}\end{array}$} & Amostra 1 & 877,52 & 838,50 & -------- & 538,43 & 493,14 & 227,04 \\
\hline & Amostra 2 & 656,34 & 765,31 & 875,32 & 458,22 & 267,85 & 546,16 \\
\hline & Amostra 3 & 871,49 & 836,95 & 793,95 & 413,63 & 581,31 & 324,06 \\
\hline & Amostra 4 & 761,31 & 673,00 & 1126,03 & 416,35 & 420,32 & 436,94 \\
\hline & Amostra 5 & 781,27 & 790,52 & 982,96 & 646,70 & 696,07 & 743,59 \\
\hline \multirow{5}{*}{ 号 } & Amostra 1 & 993,57 & 602,97 & 683,26 & 443,20 & 360,14 & 509,27 \\
\hline & Amostra 2 & 917,33 & 989,89 & 988,59 & 445,20 & 803,69 & 313,80 \\
\hline & Amostra 3 & 1223,50 & 1609,85 & 933,17 & 930,58 & 967,30 & 661,06 \\
\hline & Amostra 4 & 1164,54 & 976,63 & 956,76 & 877,02 & 1341,36 & 1448,81 \\
\hline & Amostra 5 & 806,47 & 1003,57 & 935,78 & 233,84 & 752,65 & 474,78 \\
\hline \multirow{5}{*}{ 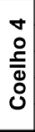 } & Amostra 1 & 703,02 & 907,23 & 1140,57 & 488,79 & 35 & 559,86 \\
\hline & Amostra 2 & 1096,39 & 1121,29 & 885,71 & 1025,17 & 453,03 & 593,04 \\
\hline & Amostra 3 & 1168,78 & 1144,00 & 1013,58 & 1235,43 & 856,71 & 735,71 \\
\hline & Amostra 4 & 1080,79 & 1092,10 & 1226,10 & 623,13 & 1142,37 & 1068,12 \\
\hline & Amostra 5 & 979,07 & 991,63 & 693,14 & 923,41 & 19 & 1387,71 \\
\hline \multirow{5}{*}{ O̊ } & Amostra 1 & 841,62 & 1010,95 & 1075,13 & 624,21 & 595,19 & 877,33 \\
\hline & Amostra 2 & 1064,16 & 999,44 & 1347,50 & 1010,67 & 785,76 & 1631,33 \\
\hline & Amostra 3 & 866,89 & 992,87 & 1333,15 & 384,07 & 446,31 & 180,43 \\
\hline & Amostra 4 & 334,98 & 745,21 & 824,39 & 933,80 & 91 & 554,42 \\
\hline & Amostra 5 & 879,74 & 836,08 & 1204,25 & 236,57 & 158,17 & 208,93 \\
\hline \multirow{5}{*}{ 号 } & Amostra 1 & 830,09 & 887,69 & 693,32 & 692,73 & 29 & 335,59 \\
\hline & Amostra 2 & 973,41 & 1211,37 & 995,82 & 498,62 & 08 & 513,00 \\
\hline & Amostra 3 & 593,75 & 840,22 & 880,83 & 764,14 & 939,33 & 706,91 \\
\hline & Amostra 4 & 1035,43 & 1119,18 & 755,55 & 673,18 & 89 & 360,79 \\
\hline & Amostra 5 & 815,26 & 897,67 & 1270,43 & 600,00 & 441,48 & 480,80 \\
\hline \multirow{5}{*}{ 龸 } & Amostra 1 & 712,04 & 710,89 & 733,11 & 505,00 &, 53 & 398,90 \\
\hline & Amo & 977,62 & 1075,58 & 1026,36 & 916,86 & 652,75 & 1182,55 \\
\hline & Amostra 3 & 1064,06 & 1148,11 & 897,23 & 1005,82 & 1395,25 & 1382,78 \\
\hline & Amostra 4 & 773,06 & 558,91 & 559,89 & 876,76 & 961,00 & 757,40 \\
\hline & Amostra 5 & 714,56 & 858,68 & 723,29 & 362,67 & 569,92 & 356,50 \\
\hline \multirow{5}{*}{ 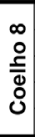 } & Amostra 1 & 737,00 & 712,12 & 645,29 & 440,61 & 388,86 & 368,05 \\
\hline & Amostra 2 & 710,80 & 1010,81 & 731,66 & 491,22 & 704,71 & 585,14 \\
\hline & Amostra 3 & 802,30 & 1218,31 & 909,50 & 405,31 & 562,38 & 471,00 \\
\hline & Amostra 4 & 864,59 & 813,17 & 965,89 & 994,26 & 951,33 & 368,24 \\
\hline & Amostra 5 & 925,25 & 894,64 & 831,55 & 96,50 & 179,67 & 410,23 \\
\hline \multirow{5}{*}{$\begin{array}{l}0 \\
\stackrel{0}{ } \\
\stackrel{\Xi}{0} \\
\stackrel{0}{0}\end{array}$} & Amostra 1 & 887,16 & 800,14 & 744,62 & 885,57 & 349,30 & 251,07 \\
\hline & Amostra 2 & 940,61 & 695,40 & 739,09 & 906,50 & 517,05 & 329,06 \\
\hline & Amostra 3 & 779,56 & 1110,70 & 732,23 & 549,80 & 675,46 & 999,19 \\
\hline & Amostra 4 & 795,97 & 1022,13 & 1074,93 & 896,83 & 764,51 & 438,58 \\
\hline & Amostra 5 & 1016,01 & 520,59 & 520,01 & 562,90 & 359,11 & 270,14 \\
\hline \multicolumn{2}{|c|}{ Coelho 10} & \multicolumn{6}{|c|}{ Excluído do estudo } \\
\hline
\end{tabular}

Tabela 1 - Valores densitométricos em UH - Grupo I

posta no local do tratamento e no local do osso normal (valor de p para o fator de interação $=0,859$ ). Além disso, o grupo 2 teve uma média estatisticamente maior que o grupo $1(p=0,034)$, tanto no local do osso normal quanto no local do tratamento, a diferença média entre os dois grupos foi de 99 unidades de medida.

\section{DISCUSSÃO}

O presente estudo objetiva utilizar a densitometria óssea para avaliar o uso das BMP, numa dosagem de 1,0 mcg, associada a hidroxiapatita como carreador, em artrodeses de coluna em coelhos.

Segundo Boden et al. ${ }^{(1)}$ e Wozney(13), três eram os tipos de biocompostos que apresentavam conhecida atividade osteoindutora, eram as rhBMPtipo2, rhBMPtipo7 ou rhOP1 e uma mistura de proteínas purificadas extraídas de osso bovino, escolhida como objeto deste estudo pela sua maior facilidade de obtenção e processamento em nosso meio.

A hidroxiapatita, utilizada como carreador, está relacionada à categoria das cerâmicas, conforme descrito por Seeherman et al. ${ }^{(12)}$, apresenta capacidade de atuar como substrato mecânico para o novo crescimento ósseo, juntamente com a propriedade de permanecer por maior tempo no sítio de ação. Segundo relatos de Helm et al. ${ }^{(6)}$, apresentam poros capazes de

\begin{tabular}{|c|c|c|c|c|c|c|c|}
\hline & & \multicolumn{3}{|c|}{ OSSO NORMAL } & \multicolumn{3}{|c|}{ ENXERTO } \\
\hline \multirow{5}{*}{$\begin{array}{l}\bar{o} \\
\stackrel{\varrho}{\Phi} \\
\text { do }\end{array}$} & Amostra 1 & 765,50 & 1312,14 & 713,70 & 409,73 & 4425,55 & 320,22 \\
\hline & Amostra 2 & 843,45 & 759,56 & 932,35 & 807,56 & 835,14 & 573,85 \\
\hline & Amostra 3 & 718,96 & 938,20 & 621,37 & 394,71 & 512,62 & 1104,24 \\
\hline & Amostra 4 & 927,64 & 1461,67 & 1158,64 & 919,71 & 671,40 & 553,40 \\
\hline & Amostra 5 & 775,51 & 462,80 & 543,62 & 391,86 & 331,03 & 692,73 \\
\hline \multirow{5}{*}{ 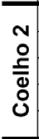 } & Amostra 1 & 1035,59 & 999,00 & 758,23 & 301,76 & 707,93 & 916,05 \\
\hline & Amostra 2 & 1156,57 & 1144,00 & 996,88 & 646,86 & 1039,13 & 695,67 \\
\hline & Amostra 3 & 988,67 & 1205,44 & 821,07 & 840,65 & 876,38 & 484,00 \\
\hline & Amostra 4 & 706,09 & 1078,96 & 725,30 & 587,86 & 534,25 & 464,65 \\
\hline & Amostra 5 & 979,95 & 1511,40 & 1098,56 & 669,57 & 469,92 & 605,38 \\
\hline \multirow{5}{*}{ 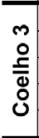 } & Amostra 1 & 932,33 & 933,77 & 857,34 & 805,52 & 364,10 & 348,04 \\
\hline & Amostra 2 & 1045,67 & 1207,06 & 1127,27 & 697,00 & 285,00 & 469,56 \\
\hline & Amostra 3 & 1009,31 & 1128,09 & 985,50 & 1057,75 & 462,97 & 509,76 \\
\hline & Amostra 4 & 1118,73 & 1172,12 & 809,10 & 1076,62 & 397,78 & 660,35 \\
\hline & Amostra 5 & 1068,56 & 1262,39 & 892,37 & 579,66 & 478,71 & 524,74 \\
\hline \multirow{5}{*}{ 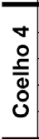 } & Amostra 1 & 1068,15 & 1123,30 & 866,83 & 819,53 & 349,89 & 167,60 \\
\hline & Amostra 2 & 1472,70 & 1145,44 & 870,38 & 754,93 & 1109,41 & 824,36 \\
\hline & Amostra 3 & 1057,92 & 739,78 & 913,00 & 1081,00 & 827,29 & 802,65 \\
\hline & Amostra 4 & 1024,57 & 645,00 & 580,69 & 369,06 & 777,97 & 555,37 \\
\hline & Amostra 5 & 1252,74 & 1137,00 & 1011,77 & 351,40 & 177,32 & 621,90 \\
\hline \multirow{5}{*}{$\begin{array}{l}10 \\
\stackrel{0}{\varrho} \\
\stackrel{\Xi}{0} \\
\stackrel{0}{0}\end{array}$} & Amostra 1 & 1082,10 & 836,66 & 900,44 & 326,02 & 484,18 & 694,00 \\
\hline & Amostra 2 & 1208,13 & 1317,65 & 1216,96 & 622,14 & 406,50 & 591,25 \\
\hline & Amostra 3 & 670,58 & 1091,00 & 737,16 & 1548,07 & 630,91 & 1055,00 \\
\hline & Amostra 4 & 1116,22 & 1175,06 & 738,38 & 984,57 & 870,18 & 1040,92 \\
\hline & Amostra 5 & 346,80 & 199,00 & 748,39 & 214,65 & 457,40 & 212,33 \\
\hline \multirow{5}{*}{$\begin{array}{l}0 \\
\circ \\
\stackrel{\bar{\Xi}}{0} \\
\stackrel{0}{0}\end{array}$} & Amostra 1 & 922,25 & 903,65 & 859,37 & 1214,29 & 679,67 & 549,37 \\
\hline & Amostra 2 & 1338,96 & 676,31 & 896,57 & 1120,13 & 776,17 & 756,83 \\
\hline & Amostra 3 & 1363,12 & 1071,12 & 743,56 & 950,29 & 1415,82 & 683,77 \\
\hline & Amostra 4 & 863,60 & 698,14 & 915,95 & 490,35 & 278,61 & 680,39 \\
\hline & Amostra 5 & 1120,44 & 1084,32 & 955,32 & 334,08 & 349,39 & 907,82 \\
\hline \multirow{5}{*}{$\begin{array}{l}\hat{0} \\
\stackrel{0}{\mathbf{c}} \\
\bar{\delta} \\
\dot{0}\end{array}$} & Amostra 1 & 1197,43 & 1129,48 & 918,43 & 1252,07 & 820,27 & 984,68 \\
\hline & Amostra 2 & 1087,33 & 1045,41 & 451,35 & 1203,61 & 1234,76 & 1239,82 \\
\hline & Amostra 3 & 1241,67 & 958,12 & 1204,44 & 557,63 & 670,38 & 1069,63 \\
\hline & Amostra 4 & 1574,53 & 1122,45 & 1627,40 & 397,62 & 1100,50 & 970,30 \\
\hline & Amostra 5 & 1043,39 & 1157,31 & 1241,70 & 208,90 & 735,85 & 938,62 \\
\hline \multicolumn{2}{|c|}{ Coelho 8} & \multicolumn{2}{|c|}{ Excluído do estudo } & \multicolumn{4}{|c|}{ Paraplegia e infecções urinárias de repetição. } \\
\hline \multirow{5}{*}{$\begin{array}{l}0 \\
\circ \\
\stackrel{\Xi}{\sigma} \\
\delta \\
0\end{array}$} & Amostra 1 & 1707,89 & 849,33 & 598,18 & 1109,29 & 868,73 & 957,00 \\
\hline & Amostra 2 & 702,93 & 764,43 & 605,87 & 936,10 & 880,89 & 974,35 \\
\hline & Amostra 3 & 1033,54 & 849,86 & 1044,79 & 1078,12 & 1152,58 & 1168,38 \\
\hline & Amostra 4 & 735,71 & 1000,12 & 356,12 & 778,82 & 1032,45 & 635,78 \\
\hline & Amostra 5 & 632,64 & 473,83 & 675,95 & 1204,65 & 1031,54 & 819,02 \\
\hline \multirow{5}{*}{$\begin{array}{l}\text { 음 } \\
\text { } \\
\frac{1}{\Phi} \\
\delta\end{array}$} & Amostra 1 & 348,94 & 479,55 & 792,77 & 889,11 & 807,93 & 1082,25 \\
\hline & Amostra 2 & 1453,44 & 890,00 & 1306,96 & 636,35 & 1003,57 & 838,38 \\
\hline & Amostra 3 & 1059,82 & 1436,50 & 1236,58 & 1394,38 & 1326,28 & 447,28 \\
\hline & Amostra 4 & 114,75 & 1185,89 & 1001,28 & 889,71 & 752,76 & 491,45 \\
\hline & Amostra 5 & 965,06 & 783,46 & 643,13 & 525,12 & 481,71 & 346,95 \\
\hline
\end{tabular}

Tabela 2 - Valores densitométricos em UH - Grupo II

reter as BMPs, dificultando assim sua absorção, logo, essas propriedades associadas conferem à hidroxiapatita grande potencial osteocondutor e, por esse motivo, foi a opção utilizada neste estudo.

Inúmeros modelos animais estão descritos na literatura. Sandhu e Khan ${ }^{(11)}$ sugerem a utilização de coelhos como modelo experimental quando forem realizadas artrodeses intertransversas, como neste estudo, além disso, esses modelos podem ser selecionados com uniformidade e permitem avaliações que não podem ser realizadas em humanos. Entretanto, Seeherman et al. ${ }^{(12)}$ alertam para o fato desses modelos não serem preditivos em humanos. Boden(2) alerta para a necessidade de criteriosa decorticação dos elementos posteriores para realização da artrodese, evitando assim pseudoartroses.

Outro fator a ser considerado, segundo Boden et al.(1), é a escolha da dosagem do biocomposto a ser utilizada; segundo recomendação de Damien et al. ${ }^{(5)}$, para modelos experimentais em coelhos devem ser utilizadas doses mínimas de 0,15 mg de BMP bovina.

Neste estudo, a opção foi por utilizar dosagem de 1,0 mg de BMP bovina, pois conforme Boden et al. (1), existe um limiar abaixo do qual essas proteínas não apresentam atividade e, ultrapassando-se esse limiar, o incremento de neoformação óssea é diretamente proporcional à quantidade de BMP implantada. 
Para a avaliação dos resultados deste trabalho foi utilizada a densitometria óssea realizada por meio de tomografia computadorizada, segundo técnica descrita por Housfield(8), por tratarse de método pouco utilizado e ainda sem precedentes de literatura que possam referendá-la como técnica de escolha.

As formas mais freqüentes de avaliação dos resultados, sugeridos por Seeherman et al. ${ }^{(12)}$, compreende a realização de radiografias em duas posições, preparações histológicas, tomografia computadorizada e ensaios biomecânicos.

Algumas técnicas não são utilizadas como rotina, como é o caso da ressonância nuclear magnética, a densitometria e até mesmo como realizado por Magim e Delling ${ }^{(10)}$, que utilizaram a cintilografia óssea com tecnésio para demonstrar as propriedades osteoindutoras das BMP tipo 7 em porcos.

Com a utilização da densitometria óssea para avaliação do uso de BMP bovina, 1,0 mg, associada a hidroxiapatita como carreador, num modelo experimental em coelhos, foi possível observar que houve diferença significativa entre o incremento ósseo obtido, quando comparado ao grupo controle.

Ressalta-se ainda, na análise do osso normal, um aumento da densidade óssea no grupo onde foi utilizado BMP, quando comparado ao grupo controle.

Densitometria também foi utilizada por Cochran et al. ${ }^{(4)}$, onde relataram resultados semelhantes aos obtidos neste estudo, evidenciando aumento da massa óssea ao utilizarem cães como modelo experimental. Utilizaram ainda implantes metálicos de titânio e dose de $0,2 \mathrm{mg} / \mathrm{ml}$ de BMP, no entanto, neste trabalho não foi realizado artrodese na coluna; os implantes foram colocados em mandíbulas, sendo assim difícil o estabelecimento de paralelos diretos de comparação de resultados.

Em trabalho realizado por Hong et al. ${ }^{(7)}$, outro modelo experimental utilizou a densitometria e avaliou o uso de BMP tipo2 para reparação de falhas ósseos previamente criados em calotas cranianas de coelhos, com utilização de duas dosagens distintas de 0,5 e 1,0 mg de BMP, porém, não encontraram diferenças significativas entre as densitometrias das duas dosagens.

Utilizando-se de modelo experimental que, em suas características, novamente difere sob alguns aspectos do utilizado neste estudo, Laffargue et al. ${ }^{(9)}$, apresentaram seus resultados, diferentes do presente, ao utilizarem coelhos como modelo experimental e realizarem a reparação de falhas ósseos em fêmures, utilizando rhBMP tipo 2 nas dosagens de $10 \mathrm{mcg}$ e 40 mcg, associada a beta-tricalciofosfato como carreador, onde as avaliações densitométricas realizadas "in vivo", não evidenciaram diferenças significativas em nenhum dos grupos avaliados.

A técnica de avaliação densitométrica descrita por Hounsfield( ${ }^{(8)}$ foi utilizada por Burkus et al. ${ }^{(3)}$, onde evidenciaram aumento significativo da densidade óssea, ao avaliarem em estudo clínico, pacientes submetidos a artrodese intersomática, fazendo uso de rhBMP tipo2, associado à gaiola de titânio, em doses variando de 4.2 a $8.4 \mathrm{mg}$ e a carreador de colágeno, quando comparado à utilização de enxerto autólogo de ilíaco. Após 24 meses, observaram aumento de 213,9 unidades Hounsfield no grupo de estudo, comparado a um acréscimo de 64,3 unidades Hounsfield para o grupo controle.

Permanecem ainda algumas dúvidas com relação à utilização de densitometria para avaliação das BMPs.

Possivelmente estudos subseqüentes poderão elucidar algumas questões, pois mesmo existindo aumento de massa óssea, este em algumas circunstâncias pode não ser quantificado pela densitometria, uma vez que para a realização do estudo, inúmeras variáveis devem ser consideradas, como a dose a ser utilizada, espécie animal a ser estudada, tipo de proteína escoIhida para o estudo, tempo de segmento, local de utilização do biocomposto, tipo de carreador, bem como a padronização das medidas densitométricas realizadas. Sendo assim, a somatória dessas variáveis faz com que inúmeros estudos sejam realizados de formas diferentes, restando poucos parâmetros diretos de comparação dos resultados.

$\mathrm{Na}$ literatura não foi encontrado estudo que apresentasse as mesmas variáveis aplicadas neste trabalho, dificultando assim, comparações dos resultados obtidos.

\section{CONCLUSÕES}

1. A densitometria óssea realizada por meio de tomografia axial computadorizada, quando utilizada para avaliar modelo experimental em coelhos submetidos à artodese de coluna lombar (L5-L6), utilizando biocomposto BMP-HAP, associado a enxerto homólogo, quando comparado ao uso de enxerto homólogo isolado, possibilitou evidenciar a presença de aumento de massa óssea.

2. O aumento de massa óssea encontrado ao avaliar-se osso normal pode estar relacionado a outros mecanismos de ação das BMPs, necessitando de estudos subseqüentes para esclarecimentos.

\section{REFERÊNCIAS BIBLIOGRÁFICAS}

1. Boden SD, Andersson GB, Anderson DG et al. Overview of bone morphogenetic proteins for spine fusion. Spine 27:S1, 2002.

2. Boden SD. Overview of the biology of lumbar spine fusion and principles for selecting a bone graft substitute. Spine 27 (16 Suppl 1):S26-31, 2002.

3. Burkus JK, Dorchak JD, Sanders DL. Radiographic assessment of interbody fusion using recombinant human bone morphogenetic protein Type 2. Lippincott Williams \& Wilkins Inc 28(4)372-377, 2003.

4. Cochran DL, Nummikoski PV, Jones AA, Makins SR, Turek TJ, Buser D. Radiographic analysis of regenerated bone around endosseous implants in the canine using recombinant human bone morphogenetic protein-2. Int $\mathrm{J}$ Oral Maxillofac Implants 12:739-748, 1997.

5. Damien CJ, Grob D, Boden SD, Benedict JJ. Purified bovine BMP extract and collagen for spine arthodesis: preclinical safety and efficacy. Spine 27 (16 Suppl 1):S50-58, 2002.

6. Helm G, Anderson DG, Andersson GB et al. Bone morphogenetic proteins: basic science. Spine 27:S9-S14, 2002.

7. Hong L, Tabata Y, Yamamoto M, Miyamoto S, Yamada K, Hashimoto N, Ikada Y. Comparison of regeneration in a rabbit skull defect by recombinant human
BMP-2 incorporated in biodegradable hydrogel and in solution. J Biomater Sci Polym Ed 9:1001-1014, 1998.

8. Hounsfield GN. Computerized transverse axial scanning (tomography): Part I. Description of system. Br J Radiol 46:1016-1022, 1973.

9. Laffargue P, Hildebrand HF, Rtaimate M, Frayssinet P, Amoureux JP, Marchandise $X$. Evaluation of human recombinant bone morphogenetic Protein-2-loaded tricalcium phosphate implants in rabbits' bone defects. Bone 25(2 Suppl):55S-58S, 1999.

10. Magin MN, Delling G. Improved lumbar vertebral interbody fusion using rhOP1. Spine 26:469-478, 2001.

11. Sandhu HS, Khan SN. Animal models for preclinical assessment of bone morphogenetic proteins in the spine. Spine 27(16 Suppl 1):S32-38, 2002.

12. Seeherman $H$, Anderson DG, Andersson GB et al. Biology of spine fusion, bone graft substitutes, and the burden of proof. Spine 27:S24-S25, 2002.

13. Wozney JM. Overview of bone morphogenetic proteins. Spine 27(16 Suppl 1) $13: S 2-8,2002$. 